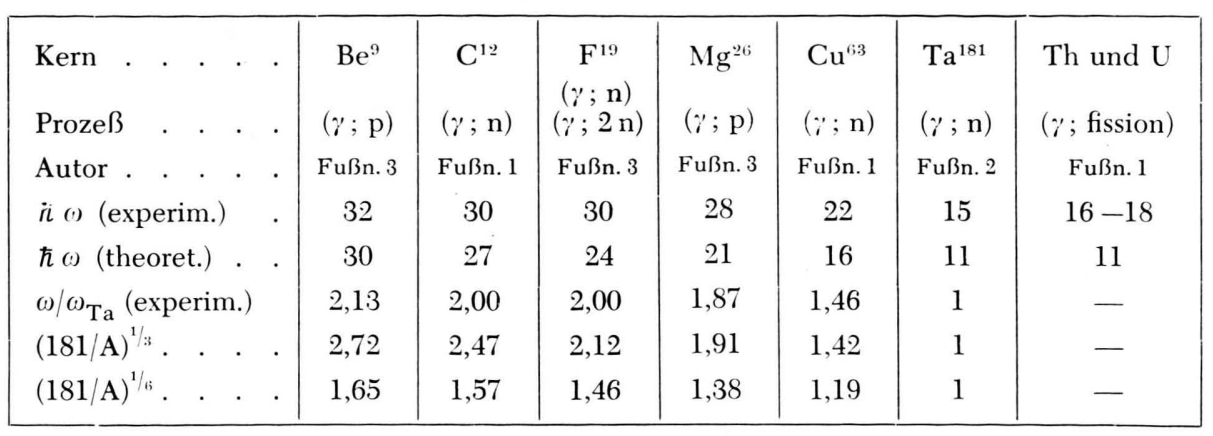

Tab. 1.

Wenn man annimmt, daß die Energie der Kollektivbewegung vor der Nucleonenemission durch die Viskosität der Kernmaterie bis zum thermischen Gleichgewicht dissipiert wird, behebt das Modell nicht die Schwierigkeit einer Erklärung dafür, daß die $(\gamma ; \mathrm{p})$ - Prozesse auch bei den schwereren Kernen noch einige Prozent der $(\gamma ; \mathrm{n})$ Prozesse ausmachen $\boldsymbol{9}$.

Eine ausführlichere Mitteilung und die Diskussion der Oszillatorstärken (Wirkungsquerschnitte, Kernstreuung) und Dämpfung (Resonanzbreite) soll a.a. O. erscheinen ${ }^{10 .}$

Hrn. H. S t e i n wed el, Heidelberg, danken wir für wertvolle Diskussionen.

9 Vgl. z. B. P. J e n s e n, Naturwiss. 35, 190 [1948].

10 H. S t e i n w e d el u. J. H. D. J e n s e n, Z. Naturforschg., in Vorbereitung.

\section{Über die statistische Berechnung des Curie-Punktes ferromagnetischer Kristallgitter}

$$
\text { Von V. Ze hler }
$$

Institut für theoret. Physik der Justus-Liebig-Hochschule Gießen

(Z. Naturforschg. 5 a, 344-345 [1950]; eingeg. am 26. Mai 1950)

Im Jahre 1937 wurde von O p e chowski 1 eine von Kramers vorgeschlagene Methode zur statistischen Berechnung thermischer und magnetischer Eigenschaften ferromagnetischer Kristallgitter für Temperaturen oberhalb des Curie-Punktes ausgearbeitet. Ausgehend vcin Heisenberg-Modell wird $F$ nach Potenzen von $1 / T$ entwickelt. Dabei bezeichnet Opechowski mit $F$ den Quoticnten aus der freien Energie pro Spin und dem Austaucchintegral I und mit $T$ das dimensionslose Verhältnis der mit cir Boltzmannschen Konstanten multiplizierten absoluten Temperatur zu $J$.

Damit ergibt sich beim kubisch-flächenzentrierten Gitter:

$$
\begin{aligned}
F=\frac{T}{2} \cdot \ln \begin{array}{r}
1-\tau^{2} \\
4
\end{array} & -3 \tau^{2}-\frac{3}{T}\left(3+20 \tau^{2}-23 \tau^{4}\right) \\
& -\frac{1}{4 T^{2}}\left(9+276 \tau^{2}-931 \tau^{4}+646 \tau^{6}\right) \ldots
\end{aligned}
$$

mit

$$
\tau=\tanh \frac{m \cdot H}{J T}
$$

( $m$ : Bohrsches Magneton, $H$ : Magnetfeld).
Die Berechnung der Koeffizienten von $T-n$ geschieht im wesentlichen durch Spurbildung mehrfacher Produkte von Pauli-Matrizen. Die praktische Berechnung wird schon für $n=4$ außerordentlich mühsam. So ist es nicht weiter verwunderlich, wenn Opechowski dabei einige Fehler unterlaufen sind, die auch in der Berichtigung 2 nicht restlos ausgemerzt wurden.

Es handelt sich, in der Bezeichnungsweise von Opechowski, um den Mittelwert des Produktes $(k l)(l m)(m k)(k p)$. Dieser muß statt $\tau^{2}$ richtig $1 / 3\left(5 \tau^{2}-4 \tau^{4}\right)$ lauten ${ }^{3}$. Damit folgt als Koeffizient von $1 / T^{3}$ in der obigen Entwicklung für $F$

$A_{4}=-{ }^{1 / 32}\left(15+9780 \tau^{2}+\right.$ höhere Potenzen von $\left.\tau\right)$.

Für die Suszeptibilität ergibt sich daraus:

$$
\text { \% } T=1+\frac{6}{T}+\frac{30}{T^{2}}+\begin{gathered}
138 \\
T^{3}
\end{gathered}+\frac{2445}{4 T^{4}}+\ldots
$$

Am Curie-Punkt wird $\chi$ stark anwachsen, und man wird mit Opechowski vermuten, daß seine ungefähre Lage durch den höchsten Wert $T_{\text {c }}$ gegeben ist, für den der Ausdruck für $1 / \chi T$ verschwindet. Durch einfache Rechnung folgt:

$$
1 / \% T=1-\frac{6}{T}+\underset{T^{2}}{6}+\stackrel{6}{T^{3}}+\frac{3}{4 T^{4}}+\ldots
$$

Indem man nach der 1., 2. oder 3. Potenz in $T$ abbricht, erhält man mit Opechowski für die Curie-Temperatur $T_{\mathrm{c}}$ nacheinander die Werte:

$$
\begin{aligned}
& \begin{array}{lll}
\text { 1. Näherung } \quad 2 \text { Näherung } \quad 3 \text { Näherung }
\end{array} \\
& T_{0}=6,0 \quad 4,73 \quad 4,26
\end{aligned}
$$

Wegen des Rechenfehlers bekam Opechowski für das Polynom 4. Grades keine Nullstelle, was ihn vermuten ließ, daß das Verfahren nicht konvergiert (S. 195, l.c. 1). Tatsächlich ergibt sich aber mit den korrigierten Werten eine Nullstelle bei $T_{\mathrm{c}}=4,25$, also fast an der gleichen Stelle wie beim Polynom 3. Grades. Das Verfahren zur Bestimmung des Curie-Punktes scheint also doch recht gut zu konvergieren.

1 W. O pe ch ow s ki, Physica 4, 181 [1937].

? W. O pe chow s ki, Physica 6, 1112 [1938].

3 Hrn. Prof. W. O pe chows ki (University of British Columbia, Vancouver, Canada) sei an dieser Stelle herzlich für die Kontrolle der Ergebnisse gedankt. 
Interessant ist eine Betrachtung der Verhältnisse beim einfach-kubischen Gitter. Man erhält:

bzw.

$$
\chi T=1+\frac{3}{T}+\frac{6}{T^{2}}+\frac{11}{T^{3}}+\frac{165}{8 T^{4}}+\ldots
$$

$$
1 / \% T=1-\frac{3}{T}+\frac{3}{T^{2}}-\frac{2}{T^{3}}+\frac{3}{8 T^{4}}+\ldots
$$

Damit ergeben sich für die Lage des Curie-Punktes folgende Werte:

$$
\begin{array}{cccc}
\text { 1. Näherung } & 2 \text {. Näherung } & \text { 3. Näherung } & \text { 4. Näherung } \\
T_{\mathrm{c}}=\quad 3,0 & - & 2 & 1,93
\end{array}
$$

Der letzte Wert stimmt überraschend gut mit dem Wert $T_{\mathrm{c}}=1,85$ überein, den kürzlich $\mathrm{W}$ e i $\boldsymbol{4}^{4,5}$ berechnet hat in Anlehnung an die Methode von Bethe ${ }^{6}$ und Peier $\mathbf{l s}^{7}$. Es ist daher zu vermuten, daß man aus der Opechowskischen Reihenentwicklung durch Abbrechen nach der vierten Potenz in $1 / T$ auch für andere Gittertypen einen brauchbaren Wert für die Curie-Temperatur wird erhalten können.

Zum Schluß möchte ich nicht versäumen, Hrn. Prof. W. D öring für die Anregungen und Ratschläge bei der Durchführung dieser Rechnungen herzlich zu danken.

4 P. R. W e i ß, Physic. Rev. 74, 1493 [1948]. [1945].

5 vgl. J. H. van Vleck, Rev. mod. Physics 17, 27

6 H. A. B e the, Proc. Roy. Soc. [London], Ser. A 150, 552 [1935].

[1936].

\section{Zur Diskussion von Kristallstrukturen ${ }^{1}$}

\section{Von Konrad Schubert}

Max-Planck-Institut für Metallforschung in Stuttgart

(Z. Naturforschg. 5 a, 345-346 [1950]; eingeg. am 3. April 1950)

Bei der Besprechung von Kristallstrukturen wird häufig der Begriff der homöopolaren Bindung benützt, der aus der Theorie des Wasserstoffmoleküls stammt und auf die durch das Austauschintegral gegebene Bindungsenergie hinweisen soll. Im Hinblick auf die Leistungsfähigkeit des Bandmodells bei der Strukturdiskussion ${ }^{2,3,4}$ erscheint es zweckmäßig, die Gittereigenfunktionen an Stelle der Molekülfunktionen hierbei heranzuziehen. Die Ortsamplitude $\psi$ des Bandmodells

$$
\psi_{k}(\boldsymbol{x})=\varphi(\boldsymbol{x}) \exp 2 \pi i \boldsymbol{k} \boldsymbol{x}\left\{\begin{array}{l}
\boldsymbol{x}=\text { Ortsvektor } \\
\boldsymbol{k}=\text { Wellenzahlvektor }
\end{array}\right.
$$

gibt nun zwar im Gitterfaktor $\varphi(x)$ einen Anhalt, Gebiete bevorzugter Anwesenheitswahrscheinlichkeit der Valenzelektronen in der Gitterzelle anzunehmen, jedoch fehlt ihr die Möglichkeit, allgemeine Ortskorrelationen der Valenzelektronen auszudrücken, weil sie als Einelektro-

1 Nach einem Vortrag, gehalten im Stuttgarter physikalischen Kolloquium am 19. Januar 1950.

2 K. S c h u be rt, Z. Metallkunde 38, 349 [1947].

3 K. S c h u bert, Z. Metallkunde 39, 88 [1948].

4 K. S chubert, Z. Metallkunde 41 [1950], im Druck. nenfunktion notwendig Unabhängigkeit von der Verteiiung der übrigen Valenzelektronen voraussetzt in dem Sinn, daß die Zweielektronenwahrscheinlichkeit ein Produkt aus zwei Einelektronenwahrscheinlichkeiten ist. Da die Ansätze von Mehrelektronenfunktionen im Gitter zur Zeit von Interesse sind $\mathbf{5 , 6 , 7}$, ist es vielleicht nützlich in dem umfassenden Experimentalmaterial, das in Gestalt erforschter Kristallstrukturen vorliegt, nach Anhaltspunkten für eine Ortskorrelation der Valenzelektronen zu suchen. Elektronenschwärme einer bestimmten (natürlich unscharfen) Schwarmgitterstruktur werden sich nicht gleichmäßig durchs Gitter bewegen, sondern gemäß einer Wahrscheinlichkeitsamplitude. Man sollte mithin Gebiete maximaler Anwesenheitswahrscheinlichkeit in der Elementarzelle annehmen an den Orten, die von den Valenzelektronen bevorzugt benutzt werden und die zur Hauptsache außerhalb der Atomrümpfe liegen. Diese Gebiete werden jedoch bei einer Fourier-Synthese nicht mehr aufgelöst, entgingen daher bislang der Aufmerksamkeit. Die ortskorrelierte Elektronenbewegung findet ihr Analogon in der kooperativen Protonendiffusion in Hydroxyden, auf die man z. B. durch eine genauere Betrachtung von Hydroxydstrukturen geführt wird ${ }^{8}$.

Im folgenden seien einige Strukturbeispiele angeführt, in denen die Annahme einer starken Ortskorrelation der Valenzelektronen zu einfachen Deutungen führt.

1. Gitter mit Elektronenschwärmen im A 2(W)-Typ.

a) Die Alkalimetalle und diejenigen Übergangsmetalle, welche im metallischen Zustand vermutlich nur 1 Elektron im Valenzband haben ${ }^{9}$, sind im A 2 (W)-Typ gebaut. Eine Überlegung von U. Dehlinger ${ }^{10}$, leicht abändernd, kann man etwa wie folgt argumentieren ${ }^{3}$. Da Elektronen gleichen Spins sich besonders stark meiden ${ }^{6}$, ist eine Schwarmstruktur im A2(W)-Typ möglich. Metalle der Valenzelektronenkonzentration 1 können ein Atomgitter bilden, das mit dem Schwarmgitter kommensurabel ist und daher einen Stabilitätsgewinn ermöglicht.

b) FeSi und mit ihm CrSi, MnSi, CoSi, RhSn, PdGa, PtGa, AuBe sind im B 20 - Typ gebaut, der eine erheblich verzerrte Abart des $\mathrm{NaCl}(\mathrm{B} 1)$-Typ darstellt. Nimmt man auf der kürzesten Verbindungslinie zwischen $\mathrm{Fe}$ und $\mathrm{Si}$ ein Elektron an und wählt dieses als Ausgangspunkt eines A 2 (W) - Elektronengitters mit den halben Elementartranslationsvektoren des $\mathrm{FeSi}$, so gelangt man zu einer plausiblen Erklärung des Typs, die keine Widersprüche zur Substanztabelle aufweist, und die gegenüber anderen Überlegungen ${ }^{11}$ durchsichtiger und anschaulicher ist.

c) Die Zellen der leichten Verbindungen $\mathrm{SiO}_{9}(\mathrm{C} 9)$ und $\mathrm{SiF}_{4}\left(\mathrm{D}_{2}\right)$ sowie der metallischen Verbindungen $\mathrm{Cu}_{2} \mathrm{O}$, $\mathrm{Ag}_{2} \mathrm{~F}$ lassen sich so in Elementarkuben der Kantenlänge

5 F. B l o c h, Z. Physik 57, 545 [1929].

6 E. Wig n e r, Physic. Rev. 46, 1002 [1934]; E. Wig ner, F. S e itz, Physic. Rev. 46, 509 [1934].

7 W. Heis en berg, Z. Naturforschg. 2 a, 185 [1947].

$8 \mathrm{~K}$. S c hubert, A. S e itz, Z. anorg. allg. Chem. 256, 226 [1948].

9 R. H o p p e, Vortrag im Strukturausschuß der Deutschen Gesellschaft für Metallkunde, Clausthal 1948.

10 U. Deh ling er, Chem. Physik der Metalle und Legierungen, Leipzig, Akad. Verlagsges. 1939.

${ }_{11}$ L. P a uling, A. M. Sold a te, Acta Cryst. 1, 212 [1948]. 